\title{
Molecular Detection of Pathogens in Negative Blood Cultures in the Lao People's Democratic Republic
}

\author{
Soo Kai Ter, ${ }^{1,2,3}$ Sayaphet Rattanavong, ${ }^{3}$ Tamalee Roberts, ${ }^{3}$ Amphonesavanh Sengduangphachanh, ${ }^{3}$ Somsavanh Sihalath, ${ }^{3}$ \\ Siribun Panapruksachat, ${ }^{3}$ Manivanh Vongsouvath, ${ }^{3}$ Paul N. Newton, ${ }^{1,3,4}$ Andrew J. H. Simpson, ${ }^{3,4}$ and Matthew T. Robinson ${ }^{3,4 *}$ \\ ${ }^{1}$ Faculty of Infectious and Tropical Diseases, London School of Hygiene and Tropical Medicine, London, United Kingdom; ${ }^{2}$ Royal Veterinary \\ College, London, United Kingdom; ${ }^{3}$ Lao-Oxford-Mahosot Hospital-Wellcome Trust Research Unit (LOMWRU), Microbiology Laboratory, Mahosot \\ Hospital, Vientiane, Lao PDR; ${ }^{4}$ Nuffield Department of Medicine, Centre for Tropical Medicine and Global Health, University of Oxford, Oxford,
} United Kingdom

\begin{abstract}
Bloodstream infections cause substantial morbidity and mortality. However, despite clinical suspicion of such infections, blood cultures are often negative. We investigated blood cultures that were negative after 5 days of incubation for the presence of bacterial pathogens using specific (Rickettsia spp. and Leptospira spp.) and a broad-range 16S rRNA PCR. From 190 samples, 53 (27.9\%) were positive for bacterial DNA. There was also a high background incidence of dengue (90/112 patient serum positive, $80.4 \%)$. Twelve samples (6.3\%) were positive for Rickettsia spp., including two Rickettsia typhi. The 16S rRNA PCR gave 41 positives; Escherichia coli and Klebsiella pneumoniae were identified in 11 and eight samples, respectively, and one Leptospira species was detected. Molecular investigation of negative blood cultures can identify potential pathogens that will otherwise be missed by routine culture. Patient management would have been influenced in all 53 patients for whom a bacterial organism was identified, and 2.3-6.1\% of patients would likely have had an altered final outcome. These findings warrant further study, particularly to determine the cost-benefit for routine use, ways of implementation, and timing of PCR for organisms such as Rickettsia and Leptospira, which are important pathogens in rural Asia.
\end{abstract}

\section{INTRODUCTION}

Bloodstream infections (BSIs) cause significant global morbidity and mortality. ${ }^{1}$ Bloodstream infections are confirmed through performing broth culture of a patient's blood for 5-7 days to detect bacteremia. This is regarded as the gold standard, ${ }^{2,3}$ and allows pathogen identification and susceptibility testing to guide treatment. Nonetheless, blood cultures are often negative. In the Lao Peoples' Democratic Republic (Lao PDR), one prospective series found $16.7 \%$ of blood cultures from inpatients were positive, yet only $10.8 \%$ were clinically relevant (the remainder is likely positive because of contamination). ${ }^{4}$ The main reasons for negativity may be that the patients had non-bacteremic illness or bacteria were present intermittently or at very low blood density. However, an additional explanation is that the causative microorganisms are delicate, fastidious, nonviable, slow growing at low densities, or uncultivable in blood culture medium. This is often seen with bacteria such as Streptococcus pneumoniae, Leptospira spp., Rickettsia spp., and Orientia spp., ${ }^{5,6}$ or when antimicrobial treatment has been started before blood collection, which may kill or inhibit pathogen growth. ${ }^{3}$ These diagnoses may therefore be missed and may be referred to as "false negatives." Timely, appropriate, and targeted antimicrobial treatment is imperative to ensure patient survival in suspected $\mathrm{BSI},{ }^{7}$ so there is a need to minimize and identify false negatives early. Previous studies have looked at specifically detecting Orientia tsutsugamushi, Rickettsia spp., and Leptospira spp. in hemoculture fluid to evaluate alternative methods of diagnosis, proving somewhat successful for O. tsutsugamushi and Leptospira spp. but not for Rickettsia spp., ${ }^{8,9}$ but no

*Address correspondence to Matthew T. Robinson, Lao-OxfordMahosot Hospital-Wellcome Trust Research Unit (LOMWRU), Microbiology Laboratory, Mahosot Hospital, Rue Mahosot, Vientiane, 01000, Lao PDR. E-mail: matthew.r@tropmedres.ac general evaluation of a broader range of bacteria was conducted. We investigated 5-day negative blood cultures for the presence of bacterial pathogens using polymerase chain reaction $(\mathrm{PCR})$.

\section{MATERIALS AND METHODS}

Ethical approval for use of blood samples was granted by the Oxford Tropical Research Ethics Committee, the University of Oxford (Oxford, United Kingdom), and the National Ethics Committee for Health Research, Lao PDR. Study patients provided written informed consent. The study was conducted between June and July 2019. Blood cultures from patients suspected of having a systemic infection were processed routinely at Mahosot Hospital Microbiology Laboratory, Vientiane, Lao PDR. Samples collected in BD BACTEC Plus Aerobic/F culture vials (Becton Dickinson, Franklin Lakes, NJ) were incubated using the BACTEC (Becton Dickinson, New Jersey) system and reported as negative after 5 days. Negative blood cultures were randomly selected from discarded culture bottles for further testing, with the only selection criterion being samples from adults ( $\geq 15$ years old). DNA extraction was performed on $0.5 \mathrm{~mL}$ of blood culture fluid using QIAamp DNA Mini Kit (Qiagen, Manchester, United Kingdom) according to Method 5 by Villumsen et al. ${ }^{10}$ Both broad and genus- or species-specific PCRs were performed (Table 1). PCR selections were based on common endemic organisms in the region. Quantitative PCRs (qPCRs) targeting Rickettsia spp. 17-kDa, ${ }^{11}$ Rickettsia typhi ompB,,$^{12}$ and Leptospira spp. rrs gene ${ }^{13}$ were performed on CFX96 Real-Time System (Bio-Rad, Bangkok, Thailand). Conventional PCRs (cPCRs) targeting O. tsutsugamushi $56-\mathrm{kDa}$ gene $^{14}$ and pan-bacterial $16 \mathrm{~S}$ rRNA $^{15}$ were performed on a C1000 Touch Thermal Cycler (Bio-Rad, Thailand); products were visualized on a $1 \%$ agarose gel. PCR products from 16S rRNApositive samples were sent for sequencing (Macrogen, Seoul, South Korea), and resulting sequences were compared against GenBank via BLASTn submission (https://blast.ncbi.nlm.nih.gov). 
TABLE 1

Details of PCRs performed

\begin{tabular}{|c|c|c|c|c|c|}
\hline Pathogen & Name & Sequence $5^{\prime}-3^{\prime}$ & Primer/Probe & Target region & $\overline{\text { Ref }}$ \\
\hline Orientia tsutsugamushi & $\begin{array}{l}56 \mathrm{kDa} \text {-RTS-8 } \\
56 \mathrm{kDa}-\mathrm{RTS}-9\end{array}$ & $\begin{array}{l}\text { AGGATTAGAGTGTGGTCCTT } \\
\text { ACAGATGCACTATTAGGCAA }\end{array}$ & $\begin{array}{l}\text { Forward } \\
\text { Reverse }\end{array}$ & $\begin{array}{l}\text { 56-kDa outer membrane } \\
\text { protein (omp) }\end{array}$ & (14) \\
\hline Rickettsia spp. & $\begin{array}{l}\text { R17K128F2 } \\
\text { R17K238R } \\
\text { R17K202TAQP }\end{array}$ & $\begin{array}{l}\text { GGGCGGTATGAAYAAACAAG } \\
\text { CCTACACCTACTCCVACAAG } \\
\text { FAM-CCGAATTGAGAACCAA } \\
\text { GTAATGC-TAMRA }\end{array}$ & $\begin{array}{l}\text { Forward } \\
\text { Reverse } \\
\text { Probe }\end{array}$ & 17-kDa surface antigen & (11) \\
\hline Rickettsia typhi & $\begin{array}{l}\text { Rt557F } \\
\text { Rt678R } \\
\text { Rt640BP }\end{array}$ & $\begin{array}{l}\text { TGGTATTACTGCTCAACAAGCT } \\
\text { CAGTAAAGTCTATTGATCCTACACC } \\
\text { FAM-CGCGAT } \\
\text { CGTTAATAGCAGCACCAGCATTA } \\
\text { TCGCG-BHQ1 }\end{array}$ & $\begin{array}{l}\text { Forward } \\
\text { Reverse } \\
\text { Probe }\end{array}$ & ompB & (12) \\
\hline Leptospira spp. & $\begin{array}{l}\text { Lepto-F } \\
\text { Lepto-R } \\
\text { Lepto-probe }\end{array}$ & $\begin{array}{l}\text { CCCGCGTCCGATTAG } \\
\text { TCCATTGTGGCCGRACAC } \\
\text { 6-FAM-CTCACCAAGGCG } \\
\text { ACGATCGGTAGC-BHQ1 }\end{array}$ & $\begin{array}{l}\text { Forward } \\
\text { Reverse } \\
\text { Probe }\end{array}$ & rrs gene & (13) \\
\hline Pan-bacterial & $\begin{array}{l}\text { 27F/N1-F } \\
518 R / N 3-R\end{array}$ & $\begin{array}{l}\text { AGAGTITGATCMTGGCTCAG } \\
\text { GTATTACCGCGGCTGCTGGCA }\end{array}$ & $\begin{array}{l}\text { Forward } \\
\text { Reverse }\end{array}$ & 16S rRNA & (15) \\
\hline
\end{tabular}

Patient records were checked, and along with other laboratory results (including PCR results for rickettsial organisms from ethylenediaminetetraacetic (EDTA) buffy coat, and Leptospira spp., dengue virus and Zika virus from serum ${ }^{11,13,16,17}$ ), discharge diagnosis and antimicrobial usage were linked to PCR results.

\section{RESULTS}

One-hundred ninety, day- 5 negative blood culture samples were studied from 189 patients. Of these, 53 (27.9\%) were positive by PCR for bacterial DNA. Twelve (6.3\%) were positive for Rickettsia spp. (qPCR quantification cycle $[\mathrm{Cq}]=34.5-38.5$ ), of which two samples were further identified as $R$. typhi $(\mathrm{Cq}=30.8-36.8)$. One sample $(0.5 \%)$ was positive for Leptospira $\mathrm{sp} .(\mathrm{Cq}=34.1)$. Panbacterial 16S rRNA cPCR gave 41 (21.6\%) positives; of these, 30 PCR products were suitable for sequencing. Escherichia coli was identified in 11 samples (identity 100\%), Klebsiella pneumoniae in eight (99-100\%), Rheinheimera spp. in five (99-100\%), Pantoea vagans in two (99-100\%), one sample was identified as Erwinia gerundensis (100\%), one as Plesiomonas shigelloides (100\%), and one as a Yersinia sp. (99\%) (Figure 1). One sample was suspected to be a polymicrobial infection from mixed sequencing results. One sample was positive for both Rickettsia spp. and $K$. pneumoniae. Orientia tsutsugamushi was not detected. Where a genus- or species-specific identification was made, 34 were classed as "potentially clinically significant," five as "not significant," and two of "uncertain clinical significance" (see Figure 1). PCR of buffy coat samples for rickettsial pathogens and Leptospira PCR (on serum) was only requested in one patient, who was negative (the blood culture fluid was also negative for any bacteria for this patient). Dengue investigations were requested for 112 of the patients in this series; 90 were positive for dengue virus RNA in serum (90/ $112,80.4 \%)$, of whom 23 (25.6\%) also had a positive bacterial PCR from the negative blood culture fluid. Bacteria identified in dengue-positive patients include Rickettsia spp. (21.7\%), Leptospira spp. (4.3\%), E. gerundensis (4.3\%), K. pneumoniae (21.7\%), E. coli (26.1\%), and P. shigelloides (4.3\%). Of the five Rickettsia spp. positives who were also positive for dengue, one patient had an admission diagnosis of "Rickettsial disease/ dengue without warning signs." However, the odds ratio (OR) of the likelihood of association between PCR-positive blood

\begin{tabular}{|c|c|c|c|c|c|c|c|c|}
\hline \multirow{2}{*}{$\begin{array}{l}\text { Clinical } \\
\text { significance }\end{array}$} & & \multicolumn{6}{|l|}{ Frequency } & \multirow[b]{2}{*}{$\%$ identity ${ }^{c}$} \\
\hline & & 0 & 4 & 6 & 8 & 10 & 12 & \\
\hline \multirow[t]{7}{*}{ Significant } & Escherichia coli & & & & & & & $100 \%$ \\
\hline & Klebsiella pneumoniae & & & & & & & $99.3-100 \%$ \\
\hline & Leptospira sp. ${ }^{\mathrm{a}}$ & & & & & & & $\mathrm{n} / \mathrm{a}$ \\
\hline & Pantoea vagans & & & & & & & $99.7-100 \%$ \\
\hline & Plesiomonas shigelloides & & & & & & & $100 \%$ \\
\hline & Rickettsia sp. ${ }^{\mathrm{a}}$ & & & & & & & $\mathrm{n} / \mathrm{a}$ \\
\hline & Rickettsia typhi & & & & & & & $\mathrm{n} / \mathrm{a}$ \\
\hline \multirow{2}{*}{$\begin{array}{l}\text { Uncertain clinical } \\
\text { significance }\end{array}$} & Erwinia gerundensis & & & & & & & $100 \%$ \\
\hline & Yersinia sp. & & & & & & & $99.7 \%$ \\
\hline Not significant & Rheinheimera sp. & & & & & & & $99.3-100 \%$ \\
\hline Unknown & No identification ${ }^{b}$ & & & & & & & \\
\hline
\end{tabular}

FIGURE 1. Numbers of organisms identified by genus- and species-specific PCRs, $16 \mathrm{~S}$ rRNA PCR, and sequencing (including percent identity match), $n=53$.

${ }^{a}$ Identification by genus- and species-specific PCRs.

b 16 S rRNA PCR-positive, but no genus or species identification could be made.

${ }^{c}$ Percent identity match by sequencing of $16 \mathrm{~S}$ rRNA PCR product. $\mathrm{n} / \mathrm{a}=$ not applicable, samples were identified by genus- or species-specific PCRs, not 16S rRNA and were not subjected to BLAST analysis. 
culture and the presence of dengue infection was not statistically significant (OR: 1.46 ; $95 \% \mathrm{Cl}$ : $0.41-6.55 ; P=0.778$ ). Of 111 patients also tested for Zika virus, all were negative, and patients were not screened for Chikungunya virus. Vientiane is not a malaria-endemic region. According to patient records, four (7.5\%) PCR-positive patients had taken ceftriaxone before sampling (organisms identified included Rickettsia sp., E. coli, and $P$. vagans) and 15 (28.3\%) had not taken antimicrobials, and for $34(64.2 \%)$ patients, this was unknown. Only two patients (one Leptospira sp.-positive and one Rickettsia sp.positive) received doxycycline. No other Rickettsia spp.positive patients received a tetracycline.

Where available, patient records were reviewed to evaluate if the patient management, diagnosis, or outcome would have been influenced or changed by the PCR result (presence/absence of bacteria) or species identification. The positive 16S PCR result would probably have influenced patient management considerations (i.e., the attending clinician would have checked current specific management or considered possible changes to management) in all 53 patients (100\%), whether or not a specific species identification had been made. Where patient management information was available, the majority of patients $(55.8 \%$, 24/43) would likely have had a change in management (i.e., given antibiotics when they originally did not receive any, or had a change in antibiotic class given). In particular, three patients identified as Rickettsia sp. positive had not been given a tetracycline; if these data had been available during patient admission, they would have had a change in management. Where a genus- or species-specific identification was made, management would likely have been influenced in $90.2 \%$ (37/41) of patients, and management likely have been changed in $69.7 \%$ (23/33) of patients. The identification of any bacteria present would have likely changed the final diagnosis in 55.8\% (24/43) of patients: $90.9 \%$ (30/33) when a genus- or species-specific identification was made. Overall, a review of patient outcomes suggested that a positive 16S rRNA cPCR result would have changed the outcome in only one patient $(2.3 \%, n=43)$, and a genus- or species-specific identification in two patients $(6.1 \%, n=33)$.

\section{DISCUSSION}

Potential bacterial pathogens were successfully identified by PCR in over $25 \%$ of blood cultures that had been reported as negative after 5 days; over $5 \%$ contained rickettsial DNA. However, this must be interpreted with caution, given the high incidence of acute dengue in this series. Any bacterial organism cultured from a sterile site (such as blood) is a potential pathogen, although in the Lao PDR, contaminants such as coagulase-negative staphylococci are frequently isolated from positive blood cultures. ${ }^{4}$ For samples containing E. coli, K. pneumoniae, R. typhi, or Leptospira spp. DNA, the findings were considered potentially clinically significant. These bacteria are unlikely contaminants, and most patients will develop febrile illnesses within days of infection. ${ }^{18,19}$ Escherichia coli and $K$. pneumoniae grow readily in blood culture media, are common isolates from blood cultures, ${ }^{20}$ and have the potential to cause serious illness. ${ }^{21}$ That these pathogens did not grow in these bottles, leading to a false negative, could be due to several reasons, such as organism death or growth inhibition from incorrect sample handling or prior antimicrobial treatment, very low circulating bacterial loads, or transient DNAemia (e.g., secondary to dengue infection). Although plausible, inappropriate handling of samples, such as storage conditions of inoculated bottles before blood culture, ${ }^{22}$ is an unlikely reason for a negative blood culture in this study as the time between sample collection and inoculating blood culture bottles is generally short in Mahosot Hospital (bottles are inoculated at the bed-side and transferred to the laboratory within a few hours). The use of incorrect blood volumes for inoculating blood culture bottles may also be discounted as the mean blood volume used was not statistically significant between PCRpositive (mean: $10.15 \mathrm{~mL}$; IQR: 9.68-10.62 mL) and PCR-negative (mean: $9.84 \mathrm{~mL}$; IQR: $9.39-10.29 \mathrm{~mL}$ ) blood cultures $(P=0.434)$. A more likely reason is a low bacterial inoculum (assuming the initial presence of viable organisms) due to antimicrobial treatment before blood culture, with the selective pressure of antimicrobials suppressing bacterial growth. ${ }^{23}$ Only $7.5 \%$ of the PCR-positive patients reported taking antimicrobials before sampling. However, given the widespread practice of self-medication with antimicrobials in the Lao PDR and ready availability of over-the-counter antimicrobials, many patients are likely to have self-treated. ${ }^{24,25}$ Patient records indicated that 25 of the PCR-positive patients had other samples taken for culture (other blood cultures, pus, urine, stool, and cerebrospinal fluid). Of these, seven patients (28\%) had positive cultures. One patient whose blood culture was PCR positive for Klebsiella pneumonia also had $K$. pneumoniae identified from two blood cultures taken within the previous 6 months. There were no other bacterial DNA PCR-positive samples which grew the same bacteria from a previous blood culture.

Although it has been shown that leptospires and rickettsial organisms such as $O$. tsutsugamushi remain viable in blood culture $^{8,9,26}$ and may even benefit diagnosis through enhancement of organism numbers, standard blood culture detection systems are unable to reveal their presence. This study has confirmed those findings, with the detection of both Leptospira spp. and Rickettsia spp., including $R$. typhi by PCR. Our results suggest that routine PCR of negative blood cultures for these organisms might be valuable in some settings.

Patients in this study had blood cultures collected because they displayed symptoms/signs of BSI. However, the relationship between the finding of bacterial DNA and the clinical manifestations cannot be proven-it could be casual and not causative. Not all patients in the study may have been genuinely bacteremic, and some of the bacterial species identified are of uncertain or doubtful clinical significance, such as Rheinheimera spp. The clinical manifestations observed in patients could be due to other non-BSI etiologies (in many cases, this may have been due to dengue) because BSI symptoms are mostly nonspecific. For patients with a PCRpositive but blood culture-negative result, interpretation has to be carried out together with the analysis of patient's clinical records to determine its clinical significance. In addition, these so-called false negatives may not be clinically significant if the bacteria detected are too low in concentration to cause disease, such as a self-limiting transient infection. ${ }^{3}$ That said, clinical review suggests that a positive PCR from negative hemoculture fluids would have influenced patient management in all 53 patients, and in $90.2 \%$ of cases when a genus or species identification was made. In addition, between $55.8 \%$ and $69.7 \%$ of patients would probably have had a change in clinical management, although only $2.3-6.1 \%$ of patients would likely have had an altered final outcome.

The finding of Rickettsia spp. and Leptospira spp. DNA in negative blood culture affirms the potential of this technique to detect such fastidious bacteria. ${ }^{8,9,25}$ The detection of Enterobacteriaceae, such as $E$. coli and $K$. pneumoniae, in negative blood cultures 
shows that a multipronged approach may improve diagnosis, even for bacteria normally considered fast growing in broth media.

These findings warrant further study (with comparison of patients who are dengue PCR positive and negative) and discussion, particularly over routine use and timing of PCR for organisms such as Rickettsia and Leptospira, which are important pathogens in Southeast Asia, and the role of dengue in bacterial infections. This will inevitably involve balancing clinical, financial, and logistical considerations, especially in low-resource settings such as the Lao PDR.

Received October 13, 2020. Accepted for publication December 20, 2020.

Published online March 1, 2021.

Acknowledgments: We would like to thank the directors and medical staff of Mahosot Hospital, and the staff of the Microbiology Laboratory and hospital wards for their support. We also thank Elizabeth Ashley for constructive comments.

Financial support: The work carried out comes under the MORU Tropical Health Network, core funded by Wellcome (grant number $106698 / Z / 14 / Z)$. Additional support was provided by the London School of Hygiene and Tropical Medicine, London, UK. P. N. N., A. J. H. S., and M. T. R. are funded through the Wellcome Trust.

Disclaimer: This study, in part, was presented as abstract (LB-5257) at the 68th Annual Meeting of the American Society of Tropical Medicine and Hygiene (ASTMH), National Harbor, MD, November 20-24, 2019. The Microbiology Laboratory, Mahosot Hospital, follows UK standards for microbiological testing, and uses CLSI guidelines. The laboratory follows ISO 15189 standards but is not internationally accredited. The laboratory participates in NEQAS (UK National External Quality Assessment Service) and the Lao PDR national EQA (External Quality Assessment) scheme.

Authors' addresses: Soo Kai Ter, Lee Kong Chian School of Medicine, Nanyang Technological University, Singapore, E-mail: tersookai@ gmail.com. Sayaphet Rattanavong, Tamalee Roberts, Amphonesavanh Sengduangphachanh, Somsavanh Sihalath, Siribun Panapruksachat, Manivanh Vongsouvath, Andrew J. H. Simpson, and Matthew T. Robinson, Lao-Oxford-Mahosot Hospital-Wellcome Trust Research Unit (LOMWRU), Microbiology Laboratory, Mahosot Hospital, Vientiane, Lao PDR, E-mails: sayaphet@tropmedres.ac, tamalee.r@tropmedres.ac, amphone@tropmedres.ac, somsavanh@ tropmedres.ac, siribun.p@tropmedres.ac, manivanh@tropmedres.ac, andrew.s@tropmedres.ac and matthew.r@tropmedres.ac. Paul N. Newton, Nuffield Department of Medicine, Centre for Tropical Medicine and Global Health, University of Oxford, Oxford, United Kingdom, E-mail: paul.newton@tropmedres.ac.

This is an open-access article distributed under the terms of the Creative Commons Attribution (CC-BY) License, which permits unrestricted use, distribution, and reproduction in any medium, provided the original author and source are credited.

\section{REFERENCES}

1. Rudd KE et al., 2020. Global, regional, and national sepsis incidence and mortality, 1990-2017: analysis for the Global Burden of Disease Study. Lancet 395: 200-211.

2. Lamy B, Dargere S, Arendrup MC, Parienti JJ, Tattevin P, 2016. How to optimize the use of blood cultures for the diagnosis of bloodstream infections? A state-of-the art. Front Microbiol 7: 697.

3. Opota O, Jaton K, Greub G, 2015. Microbial diagnosis of bloodstream infection: towards molecular diagnosis directly from blood. Clin Microbiol Infect 21: 323-331.

4. Phetsouvanh R et al., 2006. Causes of community-acquired bacteremia and patterns of antimicrobial resistance in Vientiane, Laos. Am J Trop Med Hyg 75: 978-985.

5. Fischer GW, Longfield R, Hemming VG, Valdes-Dapena A, Smith LP, 1982. Pneumococcal sepsis with false-negative blood cultures. Am J Clin Pathol 78: 348-350.
6. Chideroli RT, Goncalves DD, Suphoronski SA, Alfieri AF, Alfieri AA, de Oliveira AG, de Freitas JC, Pereira UP, 2017. Culture strategies for isolation of fastidious Leptospira serovar Hardjo and molecular differentiation of genotypes Hardjobovis and Hardjoprajitno. Front Microbiol 8: 2155.

7. Kirm TJ, Weinstein MP, 2013. Update on blood cultures: how to obtain, process, report, and interpret. Clin Microbiol Infect 19: 513-520.

8. Dittrich S et al., 2016. Survival and growth of Orientia tsutsugamushi in conventional hemocultures. Emerg Infect Dis 22: 1460-1463.

9. Dittrich $S$ et al., 2016. The utility of blood culture fluid for the molecular diagnosis of Leptospira: a prospective evaluation. Am J Trop Med Hyg 94: 736-740.

10. Villumsen S, Pedersen R, Krogfelt KA, Jensen JS, 2010. Expanding the diagnostic use of PCR in leptospirosis: improved method for DNA extraction from blood cultures. PLOS One 5: e12095.

11. Jiang J, Chan TC, Temenak JJ, Dasch GA, Ching WM, Richards AL, 2004. Development of a quantitative real-time polymerase chain reaction assay specific for Orientia tsutsugamushi. Am J Trop Med Hyg 70: 351-356.

12. Henry KM, Jiang J, Rozmajzl PJ, Azad AF, Macaluso KR, Richards AL, 2007. Development of quantitative real-time PCR assays to detect Rickettsia typhi and Rickettsia felis, the causative agents of murine typhus and flea-bome spotted fever. Mol Cell Probes 21: 17-23.

13. Smythe LD, Smith IL, Smith GA, Dohnt MF, Symonds ML, Barnett LJ, McKay DB, 2002. A quantitative PCR (TaqMan) assay for pathogenic Leptospira spp. BMC Infect Dis 2: 13.

14. Horinouchi H, Murai K, Okayama A, Nagatomo Y, Tachibana N, Tsubouchi H, 1996. Genotypic identification of Rickettsia tsutsugamushi by restriction fragment length polymorphism analysis of DNA amplified by the polymerase chain reaction. Am J Trop Med Hyg 54: 647-651.

15. Lane DJ, 1991. 16S/23S rRNA sequencing. Stackebrandt E, Goodfellow M, eds. Nucleic Acid Techniques in Bacterial Systematics. Chichester, England: John Wiley \& Sons.

16. Corman VM et al., 2016. Assay optimization for molecular detection of zika virus. Bull World Health Organ 94: 880-892.

17. Leparc-Goffart I, Baragatti M, Temmam S, Tuiskunen A, Moureau G, Charrel R, de Lamballerie X, 2009. Development and validation of real-time one-step reverse transcription-PCR for the detection and typing of dengue viruses. J Clin Virol 45: 61-66.

18. Valbuena G, 2013. Rickettsioses: pathogenesis, immunity, and vaccine development. Acta Médica Costarricense 55: 48-59.

19. De Brito T, Silva A, Abreu PAE, 2018. Pathology and pathogenesis of human leptospirosis: a commented review. Rev Inst Med Trop Sao Paulo 60: e23.

20. Ruiz-Giardin JM, Martin-Diaz RM, Jaqueti-Aroca J, Garcia-Arata I, San Martin-Lopez JV, Saiz-Sanchez Buitrago M, 2015. Diagnosis of bacteraemia and growth times. Int J Infect Dis 41: 6-10.

21. Angus DC, van der Poll T, 2013. Severe sepsis and septic shock. N Engl J Med 369: 2063.

22. Sautter RL, Bills AR, Lang DL, Ruschell G, Heiter BJ, Bourbeau $\mathrm{PP}, 2006$. Effects of delayed-entry conditions on the recovery and detection of microorganisms from BacT/ALERT and BACTEC blood culture bottles. J Clin Microbiol 44: 1245-1249.

23. Harris AM et al., 2017. Influence of antibiotics on the detection of bacteria by culture-based and culture-independent diagnostic tests in patients hospitalized with community-acquired pneumonia. Open Forum Infect Dis 4: ofx014.

24. World Health Organization, 2015. WHO Launched the First Global Antibiotic Awareness Week Campaign in Lao People's Democratic Republic. Available at: https://www.who.int/laos/news/ feature-stories/detail/who-launched-the-first-global-antibioticawareness-week-campaign-in-lao-people-s-democratic-republic. Accessed April 5, 2020.

25. Khennavong $M$ et al., 2011. Urine antibiotic activity in patients presenting to hospitals in Laos: implications for worsening antibiotic resistance. Am J Trop Med Hyg 85: 295-302.

26. Griffith ME, Horvath LL, Mika WV, Hawley JS, Moon JE, Hospenthal DR, Murray CK, 2006. Viability of Leptospira in BacT/ALERT MB media. Diagn Microbiol Infect Dis 54: 263-266. 